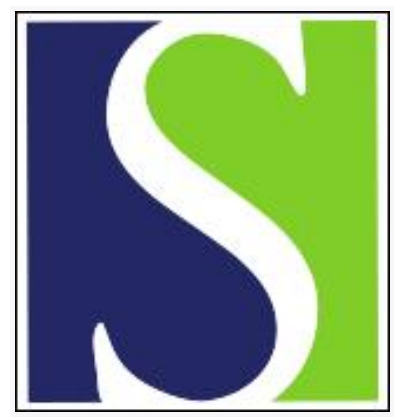

Scand J Work Environ Health 2012;38(2):134-143

https://doi.org/10.5271/sjweh.3258

Published online: 27 Oct 2011, Issue date: Mar 2012

Return to work after early part-time sick leave due to musculoskeletal disorders: a randomized controlled trial

by Viikari-Juntura E, Kausto J, Shiri R, Kaila-Kangas L, Takala E-P, Karppinen J, Miranda H, Luukkonen R, Martimo K-P

Affiliation: Finnish Institute of Occupational Health, Topeliuksenkatu 41a A, 00250 Helsinki, Finland. eira.viikari-juntura@ttl.fi

Refers to the following texts of the Journal: $2011 ; 37(2): 120-128$ 2011;37(1):1-5 2008;34(4):239-249

The following articles refer to this text: 2012;38(2):89-91; 2013;39(1):37-45; 2015;41(2):204-215; 2016;42(4):257-259; 2017;43(5):447-456; 2019;45(2):203-208; 2020;46(2):168-176; 2021;47(5):367-376

Key terms: fit note; intervention study; MSD; musculoskeletal disease; musculoskeletal disorder; occupational health practice; randomized controlled trial; RCT; return to work; RTW; sick leave; sickness absence; sickness absence; work ability

This article in PubMed: www.ncbi.nlm.nih.gov/pubmed/22033811

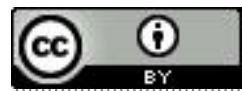




\title{
Return to work after early part-time sick leave due to musculoskeletal disorders: a randomized controlled trial
}

\author{
by Eira Viikari-Juntura, MD, PhD, ${ }^{1}$ Johanna Kausto, DDS, MSocSc, ${ }^{1,2}$ Rahman Shiri, MD, PhD, 1, 2 Leena \\ Kaila-Kangas, PhD, , 2 Esa-Pekka Takala, MD, PhD,1, 2 Jaro Karppinen, MD, PhD, 1, 2 Helena Miranda, MD, \\ PhD, ${ }^{1,2}$ Ritva Luukkonen, PhD, ${ }^{3}$ Kari-Pekka Martimo, MD, PhD ${ }^{1,2}$
}

Viikari-Juntura E, Kausto J, Shiri R, Kaila-Kangas L, Takala E-P, Karppinen J, Miranda H, Luukkonen R, Martimo K-P. Return to work after early part-time sick leave due to musculoskeletal disorders: a randomized controlled trial. Scand J Work Environ Health. 2012;38(2):134-143. doi:10.5271/sjweh.3258

Objectives The purpose of this study was to assess the effects of early part-time sick leave on return to work (RTW) and sickness absence among patients with musculoskeletal disorders.

Methods A randomized controlled trial was conducted in six occupational health units of medium- and largesize enterprises. Patients aged 18-60 years with musculoskeletal disorders $(\mathrm{N}=63)$ unable to perform their regular work were randomly allocated to part- or full-time sick leave. In the former group, workload was reduced by restricting work time by about a half. Remaining work tasks were modified when necessary, as specified in a "fit note" from the physician. The main outcomes were time to return to regular work activities and sickness absence during 12-month follow-up.

Results Time to RTW sustained for $\geq 4$ weeks was shorter in the intervention group (median 12 versus 20 days, $\mathrm{P}=0.10)$. Hazard ratio of RTW adjusted for age was 1.60 [95\% confidence interval (95\% CI) $0.98-2.63$ ] and 1.76 (95\% CI 1.21-2.56) after further adjustment for pain interference with sleep and previous sickness absence at baseline. Total sickness absence during the 12-month follow-up was about $20 \%$ lower in the intervention than the control group. Compliance with the intervention was high with no discontinuations of part-time sick leave due to musculoskeletal reasons.

Conclusions Early part-time sick leave may provide a faster and more sustainable return to regular duties than full-time sick leave among patients with musculoskeletal disorders. This is the first study to show that work participation can be safely increased with early part-time sick leave.

Key terms fit note; intervention study; musculoskeletal disease; MSD; occupational health practice; RCT; sickness absence; RTW; work ability.

Musculoskeletal disorders are a major cause of shortand long-term disability in working populations. Recent guidelines on, for example, low-back (1) and neck pain (2) and osteoarthritis (3) emphasize the importance of staying active and avoiding bed rest in preventing further disability. Employees seeking medical advice at occupational health services due to musculoskeletal disorders consider themselves more often partially able to work than unable to work (4). When work ability has been assessed as only partially reduced, the first question to be considered is whether the employee could stay at work in modified duties or work restricted hours. In the UK, following a recommendation from Dame Carol Black (5), a Statement of Fitness for Work ("fit note") was recently introduced to be used instead of the old "sick note" in the assessment of work ability (6). The fit note emphasizes remaining work capacity and provides the physician a possibility to document his or her view about the potential of phased return to work (RTW), reduced work hours, amended duties, or other workplace modifications.

A recent systematic review assessed the effects of various types of interventions on sickness absence, RTW and job retention in musculoskeletal disorders (7).

\footnotetext{
${ }^{1}$ Disability Prevention Centre, Finnish Institute of Occupational Health, Topeliuksenkatu 41 a A, 00250 Helsinki, Finland.

2 Work-related Diseases, Health and Work Ability, Finnish Institute of Occupational Health, Topeliuksenkatu 41 a A, 00250 Helsinki, Finland.

${ }^{3}$ Statistics and Health Economics, Finnish Institute of Occupational Health, Topeliuksenkatu 41 a A, 00250 Helsinki, Finland.

Correspondence to: Eira Viikari-Juntura, Finnish Institute of Occupational Health, Topeliuksenkatu 41a A, 00250 Helsinki, Finland. [E-mail: eira.viikari-juntura@ttl.fi]
} 
Thirty-four randomized controlled trials were identified (published in 1990-2010), 17 of which were targeted at the workplace, most often in combination with an exercise or behavioral intervention. The effects were in general modest: mean reduction in sickness absence being only 1.1 days per month - and even lower in higher quality studies - and similar between the different types of interventions. Van Duijn et al (8) looked at the effects of different timing of structured interventions on RTW. They modeled gained sickness absence days with differently timed interventions among populations with expected fast or slow RTW. Larger effects were not seen until 6-10 weeks of sickness absence. The authors' conclusion was, therefore, that at an early stage of sickness absence only low-cost solutions, such as accommodations of workplaces, can be expected to be cost-beneficial.

Part-time sick leave (ie, work modification with reduced work hours) offers a possibility to stay at work and be active when a disorder causes partial work disability. Partial sickness allowance is available in the Nordic countries, compensating for earnings lost during part-time sick leave. Its use has varied from one country to another (9). Studies attempting to address the effects of part-time sick leave have found somewhat inconsistent results (9) and no randomized controlled trials have been carried out (10). Partial sickness allowance was introduced in Finland in 2007. Up to 2010 and during the course of this study, the benefit could be used only after uninterrupted full-time sick leave for $>60$ working days. Occupational health professionals and other societal actors considered the lengthy period of preceding full-time sick leave to be a hindrance to early RTW.

After discussions and agreement from the Social Insurance Institution of Finland and the Ministry of Social Affairs and Health, our study group decided to carry out a randomized controlled trial with the aim of assessing the effects of early part-time sick leave on RTW and sickness absence (11). Our hypotheses were that employees, whose work time is temporarily reduced and workload adjusted during early stage of disability, will return to regular work duties faster and have less lost work days than employees on full-time sick leave.

\section{Methods}

\section{Study setting and participants}

The study was an individually randomized controlled trial in six occupational health units of medium- and large-size private or public enterprises. Employees who sought medical advice due to musculoskeletal pain in the neck or shoulder region, back, or upper or lower extremities were eligible to participate in the study. Furthermore, the symptoms and related disability had to be severe enough to justify prescription of full-time sick leave according to the prevailing practice, but still allow working reduced work hours without risk of deterioration.

A possibility to participate in the study was offered to employees $18-60$ years of age with a permanent or long-term contract, working full-time (37-38 hours per week) or nearly full-time ( $\geq 30$ hours per week), who had not been on sick leave due to their musculoskeletal health problem for $>2$ weeks during the preceding month and $>30$ days during the preceding 3 months. A further inclusion criterion was that there were no plans for surgical treatment requiring $>1$ week of sickness absence and no plans for other longer absence (longer than annual paid vacation) during 12 months after enrolment.

Subjects were excluded if they experienced acute infections, symptoms due to a major accidental injury, suspected occupational injury or disease, active inflammatory arthritis, malignant tumor diagnosed or treated during the preceding year, coexisting severe mental disorder, or pregnancy. Also subjects with very severe pain ( $>7$ on a scale from $0-10)$ or pain interfering severely with sleep ( $>7$ on a scale from $0-10)$ were excluded. This last exclusion criterion was set based on clinical experience, according to which subjects with very severe pain may not be able to work part-time.

\section{Interventions}

Patients were randomly allocated to part- or full-time sick leave. An occupational health physician recruited them for the study. In three of the six participating enterprises, the occupational physician providing services for the company carried out the recruitment; in the remaining enterprises, the occupational health services referred the potentially eligible patients to the Finnish Institute of Occupational Health.

During the initial visit, the occupational health physician informed the patient about the study and its aims. Since arrangements for part-time sick leave (and parttime work) would necessitate negotiations at the workplace, permission to contact the employee's supervisor was requested. Some of the participating companies had agreed collectively that part-time work would be arranged, while in other companies the physician confirmed this for each patient by a call to the supervisor. Thereafter informed consent was signed. The informed consent form included permission to collect sickness absence days with diagnoses from the registers of the occupational health service three months preceding and one year after recruitment. Permission was also requested to use employment data from the registers of the employers one year after recruitment. The occupa- 
tional physician carried out a symptom interview (pain intensity, pain interference with work and sleep, all assessed with a numerical scale from $0-10$, and pain in other body parts) and performed a physical examination (range of motion, selected clinical tests) according to a standardized protocol $(12,13)$. Height and weight were also inquired. The length of the subsequent sick leave was determined before randomizing the patient to the part- or full-time sick leave groups.

One day after recruitment, a researcher contacted the subjects by phone. She gave further information about the study and an internet-based questionnaire that the subjects were asked to complete after the phone call. This questionnaire inquired about sociodemographic, work (14), and lifestyle characteristics (table 1). Most subjects responded to the questionnaire within a few days and the remaining subjects after one e-mail reminder - except one who withdrew from the study shortly after enrolment. A few subjects who did not use email were interviewed over the phone.

The physicians had written instructions on recruitment and examination of patients, including a checklist of necessary procedures. They were asked to keep a list of examined patients and record reasons for refusals. The patients who declined participation were given a brief anonymous questionnaire regarding reasons for non-participation. This questionnaire was mailed to the Finnish Institute of Occupational Health.

Part- and full-time sick leave. In the part-time sick leave (intervention) group, workload was reduced by restricting work time. The recommendation was to reduce daily working time by about a half and this was achieved in most cases ( $70 \%$ of subjects). In some cases, it was difficult to arrange a half work day, and the subjects worked shorter hours on 3-4 days a week (30\% of subjects). Also, if necessary, remaining work tasks were modified to control exacerbation of activity-related symptoms. The physician gave the patient a fit note, indicating the duration of partial work disability, whether certain physical loads should be reduced, and whether any additional work modifications were deemed necessary. The patients took the fit note to their supervisor the day following their consultation.

If the worker was unable to return to full duty after the initial part-time sick leave, part-time sick leave could be continued or full-time sick leave could be prescribed based on medical assessment. Part-time sick leave could be extended up to two months, if necessary. If the worker was unable to perform part-time work duties, the part-time sick leave could be changed to full-time leave based on medical assessment. In that case, part-time sick leave could no longer be prescribed for the worker.

Full-time sick leave was prescribed for the control group.
Table 1. Characteristics of intervention and control group measured at baseline. [SD=standard deviation.]

\begin{tabular}{|c|c|c|c|c|c|c|}
\hline \multirow[t]{2}{*}{ Characteristic } & \multicolumn{3}{|c|}{$\begin{array}{l}\text { Intervention group } \\
\qquad(\mathrm{N}=31)\end{array}$} & \multicolumn{3}{|c|}{$\begin{array}{l}\text { Control group } \\
\quad(\mathrm{N}=31)\end{array}$} \\
\hline & $\%$ & Mean & SD & $\%$ & Mean & SD \\
\hline \multicolumn{7}{|l|}{ Individual characteristics } \\
\hline Gender ( $\%$ males) & 3 & & & 3 & & \\
\hline Age (years) & & 44.2 & 10.1 & & 44.4 & 10.7 \\
\hline \multicolumn{7}{|l|}{ Education } \\
\hline No vocational education & 7 & & & 13 & & \\
\hline $\begin{array}{l}\text { Basic vocational school or } \\
\text { courses }\end{array}$ & 45 & & & 37 & & \\
\hline Higher vocational school & 48 & & & 50 & & \\
\hline University level education & 0 & & & 0 & & \\
\hline
\end{tabular}

Work characteristics

In present job (years)

$12.1 \quad 9.7$

15.811 .4

Awkward trunk posture for $\geq 1$

minute at a time

Often or constantly

Lifting, carrying or pushing loads

$(16-25 \mathrm{~kg})$

$\geq 5$ times per working day

Working with hands above

shoulder level for $\geq 1$ minute at

a time

Often or constantly

Work requiring high hand grip

force

Often or constantly

Mental load at work

High or very high

Health-related characteristics

Body mass index

Current smokers

$25.4 \quad 3.6$

$27.2 \quad 5.3$

Brisk exercise ( $\geq 20$ minutes

per time) at least

2-3 times a week

$\geq 4$ times a week

32

23

Use of alcoholic beverages (at

least one glass of wine or one

bottle of beer at a time)

2-4 times a month

2-3 times a week

Musculoskeletal pain

Self-rated pain (0-10)

Pain interference with work (0-10)

Pain interference with sleep (0-10)

Onset of current problem

$<6$ weeks ago

6-12 weeks ago

$>12$ weeks ago

Primary location of

musculoskeletal pain

Back

Neck or shoulder

Upper limb

Lower limb

Musculoskeletal pain in $\geq 2$ locations

Previous sickness absence

Number of days during

previous 30 days

Median

Number of days during

previous 90 days

Median

$\begin{array}{rrrr}2.6 & 3.3 & 4.8 & 7.2 \\ 1 & & 2 & \\ & & & \\ 7.9 & 12.0 & 11.3 & 13.0 \\ 4 & & 6 & \end{array}$


In both groups, employees received their regular salary. In Finland, the employer receives compensation from the Social Insurance Institute after a full-time sickness absence has lasted ten workdays (employer period). Since early part-time sick leave was not included in the Finnish sickness benefit scheme at the time of the study, specially allocated research funds were used to partially compensate the employers for part-time sick leave exceeding the employer period.

\section{Outcome measures}

The primary outcome was time from recruitment to return to regular work activities. This was further specified as "sustained RTW for $\geq 2$ weeks" (ie, the worker continued to work without recurrent sick leave $\geq 2$ weeks after the end of part- or full-time sickness absence) and "sustained RTW for $\geq 4$ weeks". Numbers of sickness absence days (part-time and full-time) and their proportion of potential work time were calculated during the follow-up of one year. We also looked at the recurrence of sick leaves (for any cause) after the initial sick leave period and time to the first recurrence. Sickness absence days with main diagnoses were obtained from the registers of the occupational health services after the end of follow-up.

\section{Sample size}

We considered a $10 \%$ difference in the proportions of employees returning to regular work as significant $(75 \%$ and $85 \%$ RTW rates). With a power of $80 \%$ and level of significance of 0.05 and assumed drop-out rate of $10-15 \%, 600$ employees (300 in both groups) would be needed for the study. Using previous information on sickness absence in Finnish enterprises and to ensure a sufficient pool of subjects, a study base of about 30000 employees was recruited.

\section{Randomization}

Following symptom interview, physical examination, and assessment of length of sickness absence and medical treatment, physicians randomized employees to either the intervention or control group. Block randomization (block size 4) was used in order to obtain an equal size of intervention and control group for each participating physician. The allocation was performed at the Finnish Institute of Occupational Health by a statistician using a random number generator. For each physician, a researcher prepared sequentially numbered sealed opaque envelopes, containing information on the allocated group. The envelopes were kept in a locked closet in each physician's office.

\section{Blinding}

It was not possible to blind either the employee or the treating physicians to group allocation.

\section{Ethics}

The Coordinating Ethics Committee at Helsinki University Hospital approved the study.

\section{Statistical analysis}

We used descriptive statistics in the comparison of the baseline characteristics of the subjects to assess the success of the randomization.

Kaplan-Meier analyses were carried out to compare time to sustained RTW and the occurrence of recurrent sick leaves in the part- and full-time sick leave groups. We explored whether there was any clustering effect by the participating enterprises. To estimate hazard ratios (HR) for return to work, we used the Cox proportional hazard model with a cluster option. We checked and confirmed the assumption of constant HR over time for this model, and assessed the goodness-of-fit using the Grønnesby \& Borgan test (15). Separate models were run to control for variables that differed between the intervention and control group at baseline. Variables that affected the HR estimate $\geq 10 \%$ were included in the final model (16). Sensitivity analyses were performed excluding those subjects who were originally included by the examining physicians, but were found not to have fulfilled inclusion criteria based on register information (previous sickness absence $\geq 2$ weeks during the preceding month or $\geq 30$ days during the preceding 90 days; $\mathrm{N}=3$ ), initial clinical examination findings (pain intensity $\geq 8$; $\mathrm{N}=8$ ) or both $(\mathrm{N}=1)$ scrutinized at the end of follow-up. We used STATA, version 10, (StataCorp, College Station, TX, USA) for the analyses.

\section{Results}

Between November 2006 and December 2009, the study's physicians examined 120 subjects. Of these, 57 were found to be ineligible, most often because full-time sickness absence was not considered necessary or parttime working was considered hazardous to the patient (figure 1). The excluded subjects were comparable to the included subjects with regard to gender, pain level, and pain location, but they were slightly older (average age 48 versus 44 years). In total, 63 subjects were enrolled in the study, 19 of whom belonged to a pilot series that was recruited before the study was registered (21 
January 2008). Since the protocol of the pilot study was kept unaltered after this period, these individuals were included in our study sample. Even though the desired number of participants was not achieved, recruitment to our study was ended because the Finnish sickness benefit scheme was amended in the beginning of 2010, introducing early part-time sick leave similar to that used in our study.

\section{Loss to follow-up}

One subject (in the part-time sick leave group) declined participation shortly after enrolment and was excluded, leaving 62 subjects in the analysis (figure 1). The medical records were obtained for all patients. The followup (ending in December 2010) was complete up to 5 months for all 62 subjects. One patient in the control group received partial disability pension after 20 weeks and was censored thereafter. One patient in the intervention and four in the control group were censored at $26-37$ weeks due to change of employer.

\section{Non-compliance}

One patient in the intervention group was prescribed full-time sick leave due to an acute infection two days after recruitment. Another patient in the intervention group was prescribed full-time sick leave, since parttime work could not be arranged. These subjects were kept in the intervention group according to the intentionto-treat principle. None changed to full-time sick leave due to exacerbation of musculoskeletal symptoms.

\section{Patient characteristics}

The study population was strongly female-dominated (97\%) with an average age of 44 years in both the intervention and control groups. Most patients worked

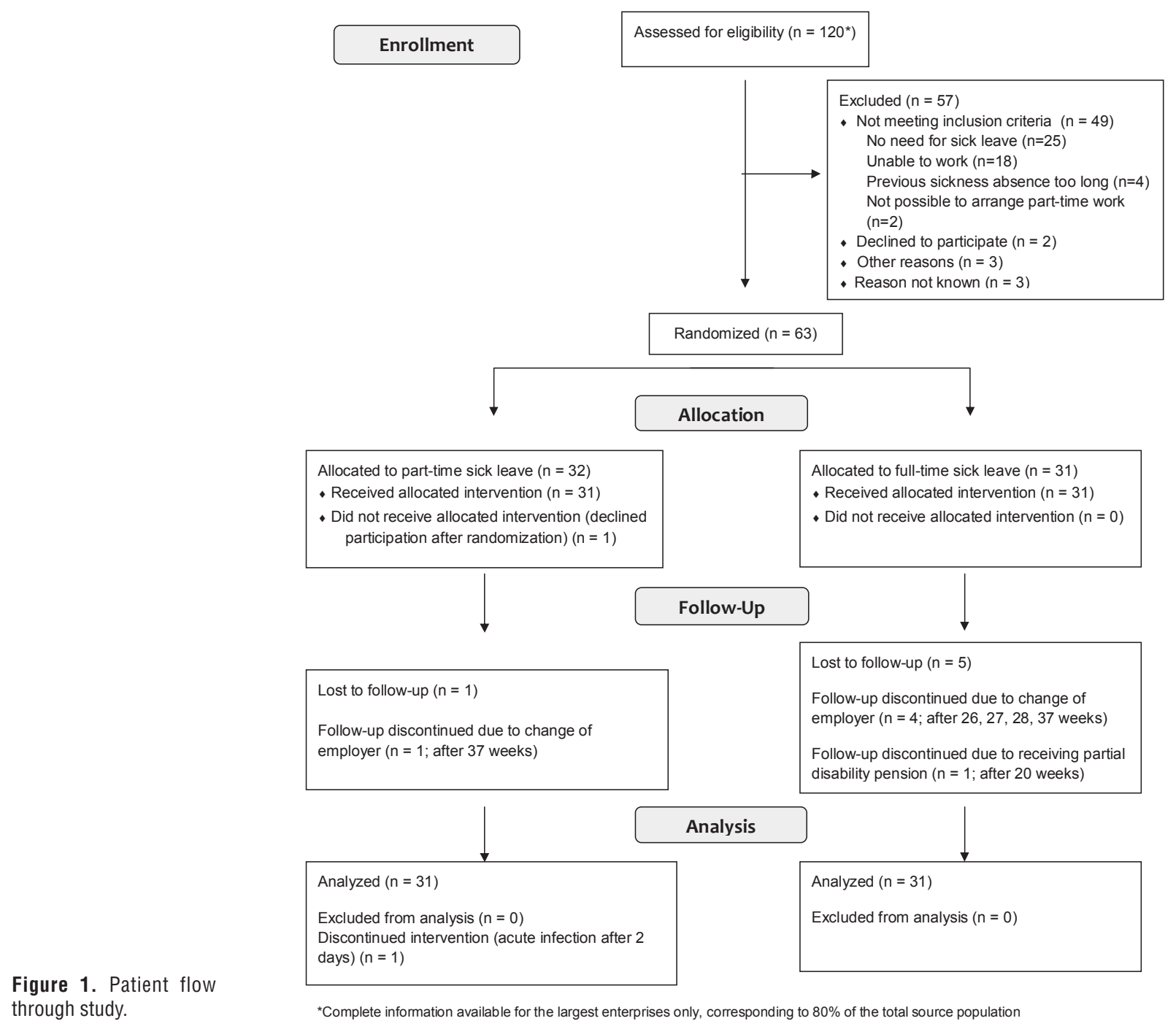


in the healthcare sector or retail trade and a minority were from the meat-processing industry or call centers. The different industries were evenly distributed between the study arms. The study groups were comparable also with regard to level of education, pain intensity, and pain interference with work. Patients in the intervention group reported more frequently heavy lifting, they were more often current smokers, had higher interference of pain with sleep, and a higher proportion of neck or shoulder problems, whereas patients in the control group had longer job tenure, higher body mass index, more chronic symptoms, more sickness absence days during 30 and 90 days preceding the baseline and higher proportion of low-back and lower-limb problems (table $1)$. Forty per cent of the subjects (13 in the intervention and 12 in the control group) had no sickness absence preceding the baseline.

\section{Work modifications}

Most patients in the part-time sick leave group performed their regular work tasks about half of the work time. One third reported a decrease in physical workload, most often in heavy lifting or other manual material handling. Five patients did tasks other than their regular work.

\section{Initial sick leave period}

The duration of the first sick leave (immediately after recruitment) was similar in the part- and full-time sick leave groups (median duration, $25^{\text {th }}$ and $75^{\text {th }}$ percentiles: 8,7 , and 11 days for the intervention and 9,7 , and 13 days for the control group, respectively).

\section{Primary outcome measures: sustained return to work and sickness absence}

Time to sustained RTW for $\geq 2$ weeks was similar in the intervention and control groups (median time: 9 days in both groups), whereas time to sustained RTW for $\geq 4$ weeks tended to be shorter in the intervention group (median 12 versus 20 days, $\mathrm{P}=0.10$ ) (table 2). When we excluded the 12 subjects who did not fulfill the inclusion criteria regarding previous sickness absence, level of pain intensity, or pain interference with sleep, there were no major changes in these results.

Patients in the part-time sick leave group returned to work earlier throughout the follow-up period (figure 2). Age-adjusted HR for RTW for $\geq 4$ weeks was 1.60 (95\% CI 0.98-2.63). The effect of the intervention differed for one large enterprise ( $\mathrm{N}=24$, age-adjusted $\mathrm{HR}$ $1.05,95 \%$ CI $0.43-2.55$ ). For the other five enterprises, the HR were close to 2 (combined HR adjusted for age $2.25,95 \%$ CI 1.10-4.59). Controlling for pain interference with sleep increased the overall age-adjusted HR by $22 \%$ and controlling for pain interference with work increased it by $9 \%$. On the other hand, controlling for previous sickness absence during the preceding 30 days decreased the HR by $11 \%$ and controlling for body mass index decreased it by $3 \%$. Overall HR, controlling for age, pain interference with sleep, and previous sickness absence, was 1.76 (95\% CI 1.21 2.56). Further adjustment for pain interference with work increased the HR to 1.84 (95\% CI $1.20-2.82)$. Controlling for the other factors, unequally distributed between the intervention and control group at baseline, did not affect the HR of RTW.

The proportion of sickness absence days decreased in both study groups during the first three months of the follow-up period and increased thereafter (table 3). The proportion of sickness absence days was lower in the intervention than the control group throughout the follow-up period. After the first week, the differences between the intervention and control group were largest between the $9^{\text {th }}$ and $26^{\text {th }}$ week. Overall, during the entire follow-up period of one year, the total number of sickness absence days was about $20 \%$ lower in the intervention than the control group.

Table 2. Time to return to work and recurrent sick leaves during the follow-up in the intervention and control group. [95\% $\mathrm{Cl}=95 \%$ confidence interval]

\begin{tabular}{|c|c|c|c|c|c|c|c|c|}
\hline & \multicolumn{4}{|c|}{ Intervention group ( $\mathrm{N}=31)$} & \multicolumn{3}{|c|}{ Control group ( $\mathrm{N}=31)$} & \multirow[b]{2}{*}{$95 \% \mathrm{Cl}$} \\
\hline & Median & $\begin{array}{l}25^{\text {th }} \\
\text { percentile }\end{array}$ & $\begin{array}{c}75^{\text {th }} \\
\text { percentile }\end{array}$ & $95 \% \mathrm{Cl}$ & Median & $\begin{array}{l}25^{\text {th }} \\
\text { percentile }\end{array}$ & $\begin{array}{c}75^{\text {th }} \\
\text { percentile }\end{array}$ & \\
\hline $\begin{array}{l}\text { Time (days from recruitment) to sustained } \\
\text { return to work for } \geq 2 \text { weeks }\end{array}$ & 9 & 6 & 22 & & 9 & 6 & 21 & \\
\hline $\begin{array}{l}\text { Time (days from recruitment) to sustained } \\
\text { return to work for } \geq 4 \text { weeks }\end{array}$ & 12 & 6 & 33 & & 20 & 8 & 35 & \\
\hline $\begin{array}{l}\text { Time (days after end of initial sick leave) } \\
\text { to the first recurrent sick leave }\end{array}$ & 29 & 4 & 85 & & 27 & 1 & 80 & \\
\hline $\begin{array}{l}\text { Average number of recurrent sick leave } \\
\text { spells per person year a }\end{array}$ & 6.5 & & & $5.1-7.9$ & 8.6 & & & $6.4-10.9$ \\
\hline
\end{tabular}




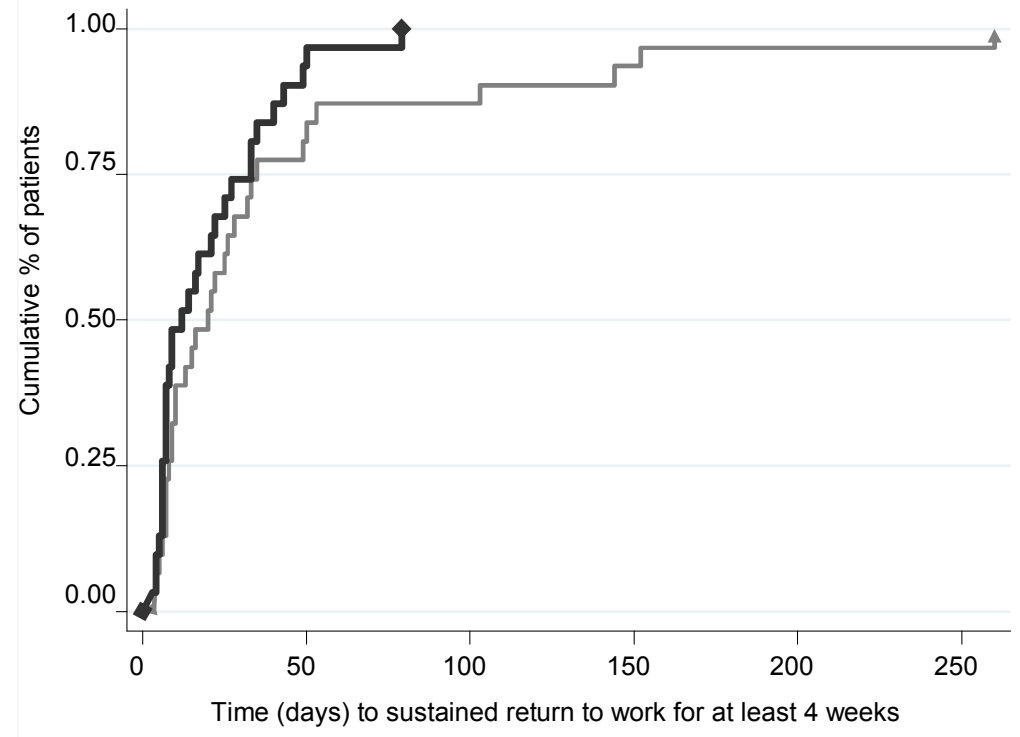

Figure 2. Return to regular work for $\geq 4$ weeks in the part- and full-time sick leave groups, unadjusted

Table 3. Total number of part- and full-time sickness absence days due to any cause during follow-up by study group (N, \% of follow-up time).

\begin{tabular}{|c|c|c|c|c|c|c|c|c|c|c|c|c|c|c|}
\hline & \multicolumn{2}{|c|}{ Week 1} & \multicolumn{2}{|c|}{ Weeks 2-3 } & \multicolumn{2}{|c|}{ Weeks 4-8 } & \multicolumn{2}{|c|}{ Weeks 9-12 } & \multicolumn{2}{|c|}{ Weeks 13-26 } & \multicolumn{2}{|c|}{ Weeks 27-52 } & \multicolumn{2}{|c|}{ Total } \\
\hline & $\mathrm{N}$ & $\%$ & N & $\%$ & $\mathrm{~N}$ & $\%$ & $\mathrm{~N}$ & $\%$ & $\mathrm{~N}$ & $\%$ & N & $\%$ & N & $\%$ \\
\hline \multicolumn{15}{|l|}{$\begin{array}{l}\text { Intervention group } \\
(\mathrm{N}=31)\end{array}$} \\
\hline $\begin{array}{l}\text { Part-time sickness } \\
\text { absence days }\end{array}$ & 189 & 44 & 88 & 10 & 100 & 5 & 47 & 3 & 0 & 0 & 0 & $0^{a}$ & 428 & 4 \\
\hline $\begin{array}{l}\text { Full-time sickness } \\
\text { absence days }\end{array}$ & 12 & 6 & 72 & 17 & 89 & 8 & 33 & 4 & 417 & 14 & 768 & 13 & 1391 & 12 \\
\hline Total & 107 & 50 & 116 & 27 & 139 & 13 & 57 & 7 & 417 & 14 & 768 & 13 & 1605 & 16 \\
\hline \multicolumn{15}{|l|}{ Control group (N=31) } \\
\hline $\begin{array}{l}\text { Full-time sickness } \\
\text { absence days }\end{array}$ & 209 & 96 & 151 & 35 & 198 & 18 & 109 & 13 & 616 & $21^{\text {a }}$ & 843 & $17^{\mathrm{b}}$ & 2126 & 20 \\
\hline
\end{tabular}

a One censored case.

${ }^{b}$ Four censored cases.

\section{Recurrence of sick leave}

Time to first recurrent sick leave was similar in both groups. However, the number of recurrent sick leaves per person year after the initial sickness absence period was about $20 \%$ lower in the intervention group (table 2).

\section{Discussion}

This randomized controlled trial on the effects of part-time sick leave suggests better work participation outcomes after part- compared with full-time sick leave among patients with musculoskeletal disorders.
Those on part-time sick leave tended to return to work sooner and have less recurrent sick leaves than those on full-time sick leave. Overall sickness absence was lower in the part-time sick leave group both in the short- and long-term perspective. Effects of sickness absence have so far been addressed in only one randomized controlled trial (17), and the present study is to our knowledge the only one published on part-time sick leave.

To measure sustained RTW, we used two variables: "RTW for $\geq 2$ weeks" was achieved in most cases after about 3 weeks on sick leave, and "RTW for $\geq 4$ weeks" after 4 weeks on sick leave. Median times to sustained RTW for $\geq 2$ and $\geq 4$ weeks were closer to each other in the part- than full-time sick leave group, indicating that 
those who once returned to work also stayed there longer in the part-time sick leave group.

The main element in the intervention was reduction of work time; most patients reduced their work time on a daily basis. Additional work modifications, such as reduction of physical workload were implemented for about a third of patients, and few did other than their regular tasks. The supervisors were contacted already at recruitment and likely had a central role in implementing the work modifications.

Exploration of the effects of the intervention in different participating enterprises revealed that the intervention was not effective in one large enterprise, contributing to more than a third of the study patients. There were no major differences in the background characteristics of the patients in this enterprise in comparison with the others that could explain the different result. At the time of our study, a major organizational turmoil was ongoing in this enterprise that may have affected the feasibility of the intervention and sickness absence behavior of the employees. However, any subgroup analyses should be interpreted with caution due to the small number of subjects in the study.

Many types of treatments have been shown to have effects on sickness absence, RTW, and job retention associated with musculoskeletal disorders $(7,18,19)$. However, recent systematic reviews (7) and economic analyses (8) have emphasized that few have proven to be cost-effective. It has been difficult to find costeffective solutions for RTW especially at the early stage of disability. In our study, most subjects on part-time sick leave were able to stay at work and avoid fulltime absence during the initial sick leave. This might bring economic benefits to an employer, but also to the employee if compensation during part-time working is higher than during sick leave.

Worsening of musculoskeletal symptoms could be a harmful effect of working with a musculoskeletal disorder. We saw no evidence of this, since no patient discontinued part-time sick leave due to this cause. There was also no difference in symptom severity between the groups during the first weeks of follow-up (data not shown).

Since the likelihood of returning to work decreases when the duration of sickness absence increases (20), we targeted the early stage of the disability, including only those who had not been off work $\geq 2$ weeks during the preceding month and $\geq 30$ days during the preceding 3 months. It is important to choose an active treatment modality - such as work participation - at an early stage to avoid iatrogenic prolongation of disability.

The fit note was one tool that was specifically developed for this study and well received in all enterprises. The form had similar elements to those in the fit note that has been adopted in use in the UK in 2010 (eg, dura- tion of disability and need for other work modifications in addition to reduction of work hours). Moreover, the most important physical workload factors were listed in the form, enabling the physician to point out whether a specific workload factor could be maintained or needed to be reduced.

\section{Comparison with other studies}

In agreement with our findings, a register-based study among subjects with a longer work disability due to various diagnoses suggests that those on part-time sick leave or with a graded RTW scheme are more likely to return to full duty than those on full-time sick leave (21). It is well-known that those on part-time sick leave present a selected group; however, in the latter study, the results seemed robust even when selection was controlled for in the analyses. In another study, part-time sick leave seemed to lead less often to full disability pension than full-time sick leave (22). In an observational study among employees with 2-6 weeks of work disability due to musculoskeletal disorders, modified work, defined either as a substantial reduction in work tasks or work hours, showed no effect on the duration of sickness absence (23).

\section{Limitations and strengths of the study}

Follow-up in the study was successful. Sickness absence information was almost complete during the first 5 months, and available for $94 \%$ of patients in the intervention and $84 \%$ in the control group at the end of the 1-year follow-up period.

Due to the nature of the intervention, it was not possible to blind either the patients or the physicians to the group allocation. However, the length of the initial sick leave was determined before randomization, guaranteeing equal length of sickness absence at baseline and making the groups comparable with regard to recurrent sickness absence during follow-up.

Inclusion and exclusion criteria were carefully considered to enable recruitment of a patient group that would benefit from part-time sick leave and to enhance high compliance with the intervention. On the other hand, care was taken in informing the workplaces to avoid an increase in those seeking medical advice due to the study. The examining physicians were also advised to keep in mind that all included patients should have a need for sickness absence based on medical assessment. Still, no need for sick leave was the most common reason for exclusion. However, medically assessed inability to work due to severity of the musculoskeletal disorder was another major reason for exclusion.

Of the two thirds of patients who were examined at the Finnish Institute of Occupational Health, very 
few declined participation. This may be because those unwilling to participate in a research study may have decided not to contact the Institute. According to the examining physicians, the proportion might have been higher among employees of those enterprises that recruited patients through their occupational health service provider; however their refusals were not systematically registered. Our sample may have therefore been selective, and we are unable to find out further information on this selection mechanism.

Almost all patients were female. The generalizability of the results may be somewhat restricted also due to the limited spectrum of industries in the study. Still, several industries and types of work were represented, such as the healthcare sector, retail trade, cleaning, meatprocessing industry, and call centre work.

To provide a study base of sufficient size, we contacted or were contacted by a total of 22 enterprises. All were visited by the researchers and the aims and the processes of the study were discussed. Only six enterprises signed the study contract. We could not initiate the project for instance in: communal daycare due to suspected problems in guaranteeing sufficient staffing throughout the day; a bus company due to anticipated problems in arranging work schedules; communal elderly care due to the occupational health service not being able to provide medical care for other than very chronic cases; and the healthcare and social sector of a smaller municipality due to resistance of the workers. Our interpretation is that the feasibility of the study was compromised by both the required arrangements for part-time sick leave in the enterprises and also the prerequisites and protocols to assess its effectiveness with a randomized controlled trial. The new law (from 2007 onwards) on part-time sick leave at a later stage of disability was considered to be an enhancing rather than hindering factor for recruitment to the study. However, when the law was amended in 2010 introducing early part-time sick leave, it was no longer possible to continue a randomized study on the effects of this benefit (24).

However, some of the participating enterprises were actively involved in the planning of the study, since they saw a clear need for agreed procedures to be able to provide modified work. They formed internal advisory groups for the study, including representatives of the employer, employees, human resource personnel, and the occupational health service. These groups fine-tuned the study protocol to the needs of the enterprise and assisted also in informing the personnel about the study.

Although our study base was very large, including more than 30000 employees, we were unable to recruit as many patients as planned. Our study had therefore low power especially to perform subgroup analyses. However, the observed difference in RTW between the intervention and comparison group was larger than anticipated. With a HR of 1.7 and drop-out rate of $10 \%$, 155 subjects would be necessary according to a power calculation (ie, considerably less than in our original study plan).

Little research evidence exists on the effects of sickness absence and modified work on health and future disability. It is often not possible to carry out a randomized trial on the effects of sickness absence. Both randomized studies and studies with other designs (eg, quasi-randomizations) could be used in future studies.

\section{Concluding remarks}

Early part-time sick leave enables patients with musculoskeletal disorders to work part-time. It may also provide a faster and more sustainable return to full duties. Taken together, work participation can be safely increased with early part-time sick leave among individuals with musculoskeletal disorders.

\section{Acknowledgements}

We thank Heidi Furu, Tuomo Pienimäki, Martti Rechardt, and Katja Ryynänen for examining patients, Merja Joutsenlahti and Eeva Hallikainen for handling patient information and other arrangements during the study, Ritva Ketola, Martta Ranta, Hilkka Riihimäki, and Tuija Toikka for participating in the earlier stages of the study, and finally participating enterprises and their occupational health personnel who referred patients to the study.

The study was supported by the Finnish Work Environment Fund (grant number 106304), the Ministry of Social Affairs and Health, and the Social Insurance Institution of Finland. The authors were independent of the funders and the funders had no role in the project. The trial is registered under Current Controlled Trials ISRCTN30911719.

The authors declare no competing interests.

\section{References}

1. van Tulder M, Becker A, Bekkering T, Breen A, del Real MT, Hutchinson A, Koes B, Laerum E, Malmivaara A. Chapter 3. European guidelines for the management of acute nonspecific low back pain in primary care. Eur Spine J 2006;15 Suppl 2:S169-91. http://dx.doi.org/10.1007/s00586-006-1071-2.

2. Binder AI. Neck pain. Clin Evid (Online) 2008(Aug 4):pii: 1103.

3. National Collaborating Centre for Chronic Conditions. Osteoarthritis: national clinical guideline for care and 
management in adults. London: Royal College of Physicians, 2008 .

4. Martimo KP, Varonen H, Husman K, Viikari-Juntura E. Factors associated with self-assessed work ability. Occup Med (Lond) 2007;57(5):380-2. http://dx.doi.org/10.1093/occmed/ kqm028.

5. Black C. Working for a healthier tomorrow. London: Crown Copyright, 2008.

6. Coggon D, Palmer KT. Assessing fitness for work and writing a "fit note". BMJ. 2010;341:c6305. http://dx.doi.org/10.1136/ bmj.c6305.

7. Palmer KT, Harris EC, Linaker C, Barker M, Lawrence W, Cooper C, Coggon D.. Effectiveness of community- and workplace-based interventions to manage musculoskeletalrelated sickness absence and job loss--a systematic review. Rheumatology (Oxford).

8. van Duijn M, Eijkemans MJ, Koes BW, Koopmanschap MA, Burton KA, Burdorf A. The effects of timing on the costeffectiveness of interventions for workers on sick leave due to low back pain. Occup Environ Med 2010;67(11):744-50. http://dx.doi.org/10.1136/oem.2009.049874

9. Kausto J, Miranda H, Martimo KP, Viikari-Juntura E. Partial sick leave--review of its use, effects and feasibility in the Nordic countries. Scand J Work Environ Health 2008;34(4):239-49.

10. Axelsson I, Marnetoft SU. Benefits and harms of sick leave: lack of randomized, controlled trials. Int J Rehabil Res;33(1):1 3. http://dx.doi.org/10.1097/MRR.0b013e32833638b9.

11. Martimo KP, Kaila-Kangas L, Kausto J, Takala EP, Ketola R, Riihimäki H, Luukkonen R, Karppinen J, Miranda H, Viikari-Juntura E. Effectiveness of early part-time sick leave in musculoskeletal disorders. BMC Musculoskelet Disord 2008;9:23. http://dx.doi.org/10.1186/1471-2474-9-23.

12. Viikari-Juntura E, Takala EP, Riihimäki H, Malmivaara A, Martikainen R, Jäppinen P. Standardized physical examination protocol for low back disorders: feasibility of use and validity of symptoms and signs. J Clin Epidemiol 1998;51(3):245-55. http://dx.doi.org/10.1016/S0895-4356(97)00266-7.

13. Viikari-Juntura E, Takala E, Riihimaki H, Martikainen R, Jappinen P. Predictive validity of symptoms and signs in the neck and shoulders. J Clin Epidemiol 2000;53(8):800-8. http:// dx.doi.org/10.1016/S0895-4356(00)00197-9.

14. The Washington State Department of Labor \& Industries. WAC 296-62-051, Ergonomics Rule (Repealed by ballot initiative12/4/03). Available online: www.lni.wa.gov/ WISHA/Rules/generaloccupationalhealth/PDFs/ ErgoRulewithAppendices.pdf (accessed 11 October 2011) 2003.
15. May S, Hosmer DW. A cautionary note on the use of the Gronnesby and Borgan goodness-of-fit test for the Cox proportional hazards model. Lifetime Data Anal 2004;10(3):283-91. http://dx.doi.org/10.1023/ B:LIDA.0000036393.29224.1d.

16. Varkevisser CM, Pathmanathan I, Brownlee AT. Designing and conducting health systems research projects. Amsterdam: KIT, 2003.

17. Borchgrevink GE, Kaasa A, McDonagh D, Stiles TC, Haraldseth O, Lereim I. Acute treatment of whiplash neck sprain injuries. A randomized trial of treatment during the first 14 days after a car accident. Spine (Phila Pa 1976) 1998;23(1):25-31.

18 Schaafsma F, Schonstein E, Ojajärvi A, Verbeek J.Physical conditioning programs for improving work outcomes among workers with back pain. Scand J Work Environ Health. 2011 Jan;37(1):1-5. http://dx. doi.org/10.5271/sjweh.3078.

19 Shiri R, Martimo KP, Miranda H, Ketola R, Kaila-Kangas L, Liira H, Karppinen J, Viikari-Juntura E. The effect of workplace intervention on pain and sickness absence caused by upper-extremity musculoskeletal disorders. Scand J Work Environ Health. 2011 Mar;37(2):120-8. http://dx.doi. org/10.5271/sjweh.3141.

20. Lötters F, Burdorf A. Prognostic factors for duration of sickness absence due to musculoskeletal disorders. Clin J Pain 2006;22(2):212-21. http://dx.doi.org/10.1097/01. ajp.0000154047.30155.72.

21. Hogelund J, Holm A, McIntosh J. Does graded return-to-work improve sick-listed workers' chance of returning to regular working hours? J Health Econ 2010;29(1):158-69. http:// dx.doi.org/10.1016/j.jhealeco.2009.11.009.

22. Kausto J, Virta L, Luukkonen R, Viikari-Juntura E, Associations between partial sickness benefit and disability pensions: initial findings of a Finnish nationwide register study. BMC Public Health 2010;10:361. http://dx.doi. org/10.1186/1471-2458-10-361.

23. Van Duijn M, Lötters F, Burdorf A. Influence of modified work on return to work for employees on sick leave due to musculoskeletal complaints. J Rehabil Med. 2005 May;37(3):172-9.

24. Maeland S, Magnussen LH, Eriksen HR, Malterud K. Why are general practitioners reluctant to enrol patients into a RCT on sick leave? A qualitative study. Scand J Public Health 2011 Sep 29. [Epub ahead of print].

Received for publication: 19 July 2011 